\title{
Comparing Leaf and Root Insertion
}

\author{
Jaco Geldenhuys, Brink van der Merwe \\ Computer Science Division, Department of Mathematical Sciences, Stellenbosch University, Private Bag X1, \\ 7602 Matieland, SOUTH AFRICA
}

\begin{abstract}
We consider two ways of inserting a key into a binary search tree: leaf insertion which is the standard method, and root insertion which involves additional rotations. Although the respective cost of constructing leaf and root insertion binary search trees trees, in terms of comparisons, are the same in the average case, we show that in the worst case the construction of a root insertion binary search tree needs approximately $50 \%$ of the number of comparisons required by leaf insertion.
\end{abstract}

KEYWORDS: Binary search trees, leaf insertion, root insertion.

\section{INTRODUCTION}

Binary search trees have been used in computer science for about fifty years, but as Jonassen and Knuth noted [5], even a simple question about these data structures may require an unexpectedly nontrivial analysis to answer. In this paper we consider the relative merits of leaf insertion and root insertion, two ways of constructing (nonbalanced) binary search trees.

Leaf insertion is the "common" method of adding a key to a binary search tree. The result of inserting a key $a$ into an empty tree, is a tree with a root node with $a$ as its key and empty left and right subtrees. If $a$ is inserted into a non-empty tree, the result is the original tree, but with $a$ inserted recursively into the left (or right) subtrees, depending on whether it is smaller (or larger) than the root key.

Root insertion is similar to leaf insertion, except that after a key $a$ has been inserted, its node $n$ is moved to the root of the tree through a series of rotations. There are two kinds of rotations, as shown in Figure 1. If $n$ is the left child of its parent, the parent is right rotated. Similarly, if $n$ is the right child of its parent, the parent is left rotated. The construction of a four element tree is shown in Figure 2. For each root insertion, the number of comparisons required is equal to the number of rotations needed to move the new key to the root.

Email: Jaco Geldenhuys jaco@cs.sun.ac.za, Brink van der Merwe abvdm@cs.sun.ac.za

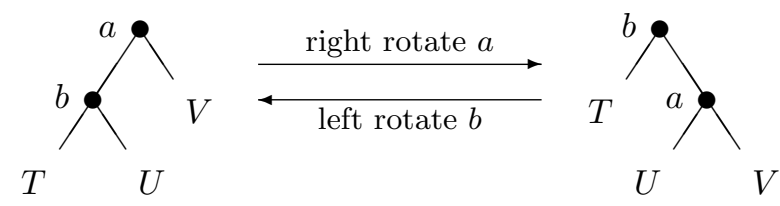

Figure 1: Right and left rotation

A comparison moves the key, to be inserted, down one level, while each rotation moves the key back up one level. Rotations are of course well-known from their use in AVL and splay trees. See for example [1] and [8].

It is important to note that rotations preserve the inorder numbering of a tree. In other words, rotation in a binary search tree produces another binary search tree.

To build an $n$-element tree, root insertion requires precisely $n-1$ comparisons (compared to leaf insertion's $\Theta(n \log n))$ in the best case, when the keys are arranged in ascending or descending order. This raises the question of whether it is possible that root insertion also has better average-case and worst-case behaviour (at least in terms of number of comparisons). Our main goal is to obtain the explicit value for $W_{n}^{r}$ in the following table.

\begin{tabular}{lcc}
\hline & $\begin{array}{c}\text { Leaf } \\
\text { insertion }\end{array}$ & $\begin{array}{c}\text { Root } \\
\text { insertion }\end{array}$ \\
\hline Best & $2+\lfloor\log n\rfloor(n+1)-2^{\lfloor\log n\rfloor+1}$ & $n-1$ \\
Ave. & $2(n+1) H_{n+1}-4 n-2$ & $A_{n}^{r}$ \\
Worst & $n(n-1) / 2$ & $W_{n}^{r}$ \\
\hline
\end{tabular}

We denote by $H_{n}$ the $n$-th Harmonic number and $\log n$ is taken base 2 . The average case for leaf 


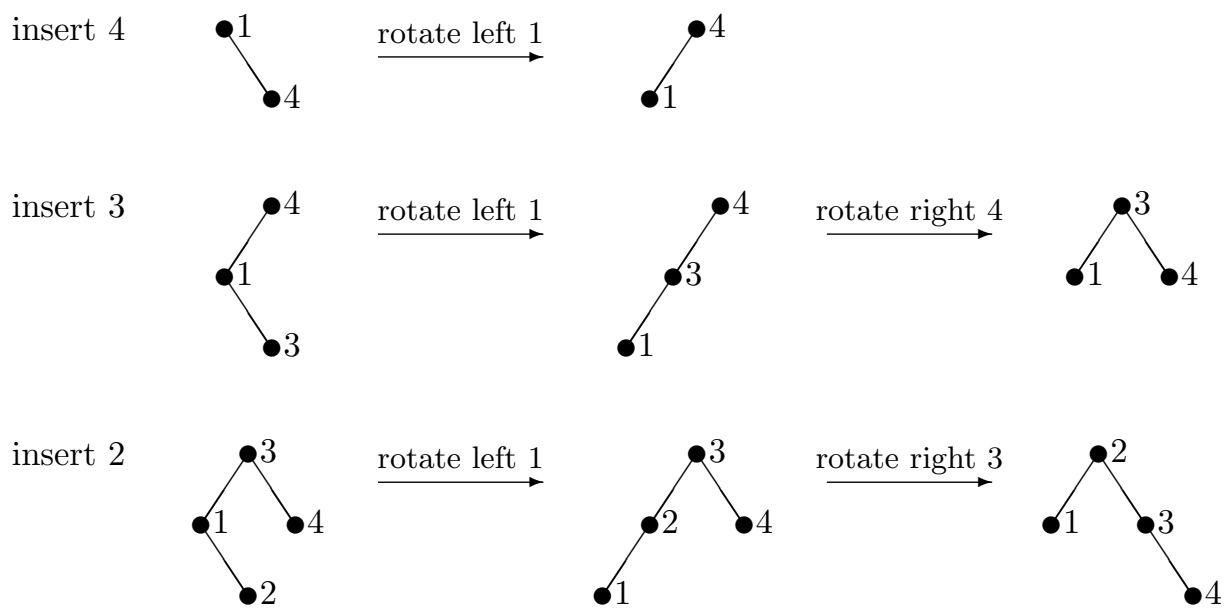

Figure 2: Construction of a four element binary search tree by root inserting 1, 4, 3, and 2; the total number of comparisons (or rotations) is equal to 5 .

insertion is obtained from [3, p. 247], and the best case for leaf insertion by simplifying the expression $\sum_{i=1}^{n}\lfloor\log n\rfloor$. It turns out that

$$
W_{n}^{r}=n(n / 4+1)-2-\alpha,
$$

where $\alpha=0$ for $n$ even, and $\alpha=1 / 4$ for $n$ odd. Thus the worst-case cost for root insertion is just a little more than half the worst-case cost of leaf insertion, for $n>249\left(0.50<W_{n}^{r} /(n(n-1) / 2)<\right.$ 0.51 if $n>249)$. For the average case we have that

$$
A_{n}^{r}=2(n+1) H_{n+1}-4 n-2,
$$

just as in the case for leaf insertion.

Interestingly, the best-case input for root insertion corresponds to the worst-case input for leaf insertion. There is another interesting correspondence between the trees constructed by root and leaf insertion: The tree built by leaf insertion from a list of keys $a_{1}, a_{2}, \ldots, a_{n}$, is identical to the tree built by root insertion of $a_{n}, a_{n-1}, \ldots, a_{1}$. It is interesting to note that identical trees are obtained if root insertion is used to build binary search trees from the sequences $1,2,4,3$ and $1,4,2,3$, but that the number of comparison required to build these trees, are not the same. This is of course in sharp contrast with leaf insertion where the number of comparisons required to construct a binary search tree, is equal to the sum of the root to node path lengths of all the nodes in the binary tree.

It should be noted that root insertion is more efficient than leaf insertion, in cases where tree searches often refer to recently inserted keys. Rotations may also be used to move an element to the root after a successful search.
After introducing the necessary notation in Section 2, we prove in Section 3 that the tree built by leaf insertion from $a_{1}, a_{2}, \ldots, a_{n}$, is identical to the tree built by root insertion from $a_{n}, a_{n-1}, \ldots, a_{1}$. The worst case performance of root insertion is analysed in Section 4, and experimental results and conclusions are presented in Sections 5 and 6, respectively.

Our interest in the performance of root insertion stems from [7, Exercise 12.85], where the reader is asked to compute $W_{10}^{r}$, and from [9], where the result was verified by exhaustive search, for sequences of length 10 and smaller. The version of root insertion described above, may more precisely be referred to as bottom-up root insertion. In [9], top-down root insertion is considered and it is shown that:

1. The tree built by leaf insertion from $a_{1}, a_{2}, \ldots, a_{n}$ is identical to the tree built by top-down root insertion from $a_{n}, a_{n-1}, \ldots, a_{1}$;

2. $A_{n}^{r}=2(n+1) H_{n+1}-4 n-2$ for top-down root insertion.

In Section 3 we show that the trees constructed, and the number of comparisons required, for top-down and bottom-up root insertion are always equal.

According to Knuth [6], leaf insertion was discovered independently by several people during the 1950s. He cites an unpublished memorandum by A. I. Dumey dated August 1952, but the first published algorithms appeared in the early 1960s [2, 4]. The rotation operation was first proposed by Adelson-Velsky and Landis in their 1962 paper on balanced trees [1]. 


\section{NOTATION}

Let $K$ be an arbitrary set of keys with a corresponding total ordering $\prec$. A sequence $s=a_{1} a_{2} \ldots a_{n}$ is considered as a specific permutation of the $n$ distinct keys $a_{1}, \ldots a_{n}$. The length $n$ of $s$ is denoted by $|s|$, and the reverse sequence $a_{n} a_{n-1} \ldots a_{1}$ by $\operatorname{rev}(s)$.

By $T_{K}$ we denote the set of binary trees over $K$, which are defined inductively as follows. We have that $t \in T_{K}$ if and only if

1. $t$ is the empty tree $\perp$, or

2. $t=a[u, v]$, where $u, v \in T_{K \backslash\{a\}}$ and $a \in K$.

The following attributes will play an important role in the remainder of this paper.

\begin{tabular}{ccc}
\hline & $t=\perp$ & $t=a[u, v]$ \\
\hline$K(t)$ & undef. & $a$ \\
$L(t)$ & undef. & $u$ \\
$R(t)$ & undef. & $v$ \\
$H(t)$ & 0 & $1+\max \{H(u), H(v)\}$ \\
keys $(t)$ & $\emptyset$ & $\{a\} \cup \operatorname{keys}(u) \cup \operatorname{keys}(v)$ \\
leaves $(t)$ & $\emptyset$ & $\{a\}$ if $u=v=\perp$, \\
& & else leaves $(u) \cup$ leaves $(v)$ \\
\hline
\end{tabular}

The set of binary search trees is a subset of $T_{K}$ denoted by $B_{K}$, and $t \in B_{K}$ if and only if $t \in T_{K}$ and

1. $t=\perp$, or

2. $t=a[u, v]$ where $u, v \in B_{K}$ and $b \prec a$ for all $b \in \operatorname{keys}(u)$ and $a \prec c$ for all $c \in \operatorname{keys}(v)$.

Since we deal exclusively with binary search trees from now on, we shall refer to them simply as trees. Note that we do not consider trees with duplicate keys.

We are now ready to formally define leaf insertion, bottom-up root insertion, and top-down root insertion.

Definition 2.1 Let $t \in B_{K}$ and $a \in K$ with $a \notin$ keys $(t)$. The tree that results from the leaf insertion of a into $t$ is

$$
L I(t, a)= \begin{cases}a[\perp, \perp] & \text { if } t=\perp, \\ K(t)[L I(L(t), a), R(t)] & \text { if } a \prec K(t), \\ K(t)[L(t), L I(R(t), a)] & \text { otherwise. }\end{cases}
$$

Let $s=a_{1} a_{2} \ldots a_{n}$. The leaf insertion tree constructed from $s$ is given by

$$
L T(s)= \begin{cases}\perp & \text { if }|s|=0 \\ \operatorname{LI}\left(\operatorname{LT}\left(a_{1} a_{2} \ldots a_{n-1}\right), a_{n}\right) & \text { otherwise. }\end{cases}
$$

Definition 2.2 Let $t \in B_{K}$ and $a \in K$ with $a \notin$ keys $(t)$. The tree that results from the bottom-up root insertion of a into $t$ is

$$
\begin{aligned}
& R I(t, a)= \\
& \left\{\begin{array}{cl}
a[\perp, \perp] & \text { if } t=\perp, \\
K(u)[L(u), K(t)[R(u), R(t)]] & \text { if } a \prec K(t), \\
\text { where } u=R I(L(t), a) & \\
K(u)[K(t)[L(t), L(u)], R(u)] & \text { otherwise. } \\
\text { where } u=R I(R(t), a) &
\end{array}\right.
\end{aligned}
$$

Let $s=a_{1} a_{2} \ldots a_{n}$. The bottom-up root insertion tree constructed from $s$ is given by

$$
R T(s)= \begin{cases}\perp & \text { if }|s|=0, \\ R I\left(R T\left(a_{1} a_{2} \ldots a_{n-1}\right), a_{n}\right) & \text { otherwise. }\end{cases}
$$

Let $l$ are $r$ be symbols that are not in $K$. Denote by $T_{K}[l, r]$ the trees in $T_{K \cup\{l, r\}}$, with $K(t) \in K$, exactly one leaf node in $L(t)$ labeled by $l$, exactly one leaf node in $R(t)$ labeled by $r$, and all other nodes are labeled by keys in $K$. Let $t_{1}, t_{2} \in T_{K \cup\{l, r\}}$ and $t \in T_{K}[l, r]$. Then $t \llbracket t_{1}, t_{2} \rrbracket$ denotes the tree obtained by replacing the node labeled by $l$ with $t_{1}$, and the node labeled by $r$ with $t_{2}$. Using the same notation as for trees in $T_{K}$, we denote by $a\left[t_{1}, t_{2}\right]$, with $a \in K, t_{1}, t_{2} \in T_{K \cup\{l, r\}}$, the tree $t$ in $T_{K}[l, r]$ with $R(t)=a, L(t)=t_{1}$ and $R(t)=t_{2}$. We denote by $B_{K}[l, r]$ all trees $t \in T_{K}[l, r]$, such that $t \llbracket \perp, \perp \rrbracket \in$ $B_{K}$.

Definition 2.3 Let $t \in B_{K}$ and $a \in K$ with $a \notin$ keys $(t)$. The tree that results from the top-down root insertion of a into $t$ is given by

$$
R I^{t o p}(t, a):=R I^{\tau}(t, a[l, r]),
$$

where $R I^{\tau}\left(t_{1}, t_{2}\right) \in B_{K}$, for $t_{1} \in B_{K}$ and $t_{2} \in$ $B_{K}[l, r]$, is defined inductively on the height of $t_{1}$, as follows.

$$
\begin{aligned}
& R I^{\tau}\left(t_{1}, t_{2}\right)= \\
& \left\{\begin{array}{cl}
t_{2} \llbracket \perp, \perp \rrbracket & \text { if } t_{1}=\perp, \\
R I^{\tau}\left(L\left(t_{1}\right), t_{2} \llbracket l, v \rrbracket\right) & \text { if } K\left(t_{2}\right) \prec K\left(t_{1}\right), \\
\text { where } v=K\left(t_{1}\right)\left[r, R\left(t_{1}\right)\right] \\
R I^{\tau}\left(R\left(t_{1}\right), t_{2} \llbracket u, r \rrbracket\right) & \text { otherwise. } \\
\text { where } u=K\left(t_{1}\right)\left[L\left(t_{1}\right), l\right]
\end{array}\right.
\end{aligned}
$$

Let $s=a_{1} a_{2} \ldots a_{n}$. The top-down root insertion tree constructed from $s$ is given by

$$
\begin{aligned}
& R T^{\text {top }}(s)= \\
& \begin{cases}\perp & \text { if }|s|=0, \\
R I^{\text {top }}\left(R T^{\text {top }}\left(a_{1} \ldots a_{n-1}\right), a_{n}\right) & \text { otherwise. }\end{cases}
\end{aligned}
$$

To illustrate top-down root insertion, we consider $R I^{\text {top }}(3[1[\perp, \perp], 4[\perp, \perp]], 2)$. We have that

$$
\begin{aligned}
& R I^{\text {top }}(3[1[\perp, \perp], 4[\perp, \perp]], 2) \\
= & R I^{\tau}(3[1[\perp, \perp], 4[\perp, \perp]], 2[l, r]) \\
= & R I^{\tau}(1[\perp, \perp], 2[l, 3[r, 4[\perp, \perp]]]) \\
= & R I^{\tau}(\perp, 2[1[\perp, l], 3[r, 4[\perp, \perp]]]) \\
= & 2[1[\perp, \perp], 3[\perp, 4[\perp, \perp]]]
\end{aligned}
$$


The definitions of top-down and bottom-up root insertion, are formal versions of the pseudocode for root insertion as described in [7] and [9], respectively.

The difference between leaf, and for example top-down root insertion, looks formidable when comparing Definitions 2.1 and 2.2, but as explained in the introduction, for $t \in B_{K}$ and $a \in K, L I(t, a)$ and $R I(t, a)$ require the same number of comparisons. From Definition 2.3, it can also be shown that $L I(t, a)$ and $R I^{t o p}(t, a)$ require the same number of comparisons. From now on we denote by $C(t, a)$ the number of comparisons required for $L I(t, a)$, $R I(t, a)$ or $R I^{t o p}(t, a)$. We can now define the cost required to build a binary tree with leaf insertion, bottom-up, and top-down root insertion respectively.

Definition 2.4 For $s=a_{1} a_{2} \ldots a_{n}$, let $\bar{s}=$ $a_{1} a_{2} \ldots a_{n-1}$. The cost to construct a tree for $s$ with leaf insertion, is denoted by $L C(s)$ and defined inductively as follows.

$$
L C(s)= \begin{cases}0 & \text { if }|s|=1, \\ C\left(L T(\bar{s}), a_{n}\right)+L C(\bar{s}) & \text { if }|s|>1 .\end{cases}
$$

Similarly, the cost to construct a tree for $s$ with bottom-up root insertion, is denoted by $R C(s)$ and defined inductively as follows.

$$
R C(s)= \begin{cases}0 & \text { if }|s|=1, \\ C\left(R T(\bar{s}), a_{n}\right)+R C(\bar{s}) & \text { if }|s|>1 .\end{cases}
$$

Finally, the cost to construct a tree for $s$ with topdown root insertion, is denoted by $R C^{\text {top }}(s)$ and defined inductively as follows.

$$
\begin{aligned}
& R C^{t o p}(s)= \\
& \begin{cases}0 & \text { if }|s|=1, \\
C\left(R T^{t o p}(\bar{s}), a_{n}\right)+R C^{t o p}(\bar{s}) & \text { if }|s|>1 .\end{cases}
\end{aligned}
$$

\section{PROPERTIES OF ROOT INSERTION}

The main results in this section state that the leaf insertion tree of a sequence $s$ is identical to the bottom-up root insertion tree of $\operatorname{rev}(s)$, and that top-down and bottom-up root insertion are equivalent in terms of trees constructed and number of comparisons required. In the first result, we show that if we use Definition 2.2, from the previous section, for top-down root insertion, then the inserted key do indeed end up at the root of the newly constructed tree.

Lemma 3.1 Let $t \in B_{K}$ and $a \in K$ with $a \notin$ keys $(t)$. Then $K(R I(t, a))=a$.
Proof (By strong induction over tree heights.) Base case: Let $t=\perp$ so that $H(t)=0$. Then $K(R I(t, a))=K(R I(\perp, a))=K(a[\perp, \perp])=a$.

Induction step: Assume that the claim holds for all trees of height less than $n$. In other words, $K(R I(t, a))=a$ for all $t \in B_{K}$ such that $H(t)<n$. Now consider $t=b[u, v] \in B_{K}$, where $H(t)=n$. This means that $H(u)<n$ and $H(v)<n$. If $a \prec b$, then

$$
\begin{aligned}
& K(R I(t, a)) \\
& =K(R I(b[u, v], a)) \\
& =K(K(w)[L(w), b[R(w), v]]) \quad w=R I(u, a) ; a \prec b \\
& =K(a[L(w), b[R(w), v]]) \quad \text { induc., } H(u)<n \\
& =a
\end{aligned}
$$

and similarly if $b \prec a$.

The result stated in the next lemma will be used in an inductive way in order to obtain Theorem 3.3.

Lemma 3.2 Let $t \in B_{K}$ and $a, b \in K$ with $a, b \notin$ keys $(t)$, such that $a \neq b$. Then $\operatorname{RI}(L I(t, b), a)=$ $L I(R I(t, a), b)$.

Proof (By strong induction over tree heights.) Base case: Let $t=\perp$ and therefore $H(t)=0$. If $a \prec b$, then

$$
R I(L I(t, b), a)=R I(b[\perp, \perp], a)=a[\perp, b[\perp, \perp]]
$$

and

$$
L I(R I(t, a), b)=L I(a[\perp, \perp], b)=a[\perp, b[\perp, \perp]],
$$

and similarly, if $b \prec a$.

Induction step: Assume that the claim holds for trees of height less than $n$. In other words, $L I(R I(t, a), b)=R I(L I(t, b), a)$ for all $t \in B_{K}$ such that $H(t)<n$. Now consider $t=c[u, v] \in B_{K}$ where $c \in K$ and $H(t)=n$. This means that $H(u)<n$ and $H(v)<n$. There are six orderings of $a, b$, and $c$ to consider. We assume that $a \prec b \prec c$. The induction step for the other cases can be obtained by similar arguments.

$$
\begin{array}{ll}
R I(L I(t, b), a) & \\
=R I(L I(c[u, v], b), a) & \\
=R I(c[L I(u, b), v], a) & b \prec c \\
=a\left[L\left(w^{\prime}\right), c\left[R\left(w^{\prime}\right), v\right]\right] & w^{\prime}=R I(L I(u, b), a) ; a \prec c
\end{array}
$$

and

$$
\begin{aligned}
& L I(R I(t, a), b) \\
& =L I(R I(c[u, v], a), b) \\
& =L I(a[L(w), c[R(w), v]], b) \quad w=R I(u, a) ; a \prec c \\
& =a[L(w), L I(c[R(w), v], b)] \quad a \prec b \\
& =a[L(w), c[L I(R(w), b), v]] \quad b \prec c
\end{aligned}
$$


Suppose that $w=R I(u, a)=d[x, y]$. By Lemma 3.1, $K(R I(u, a))=a$, hence $d=a$. Thus

$$
\begin{aligned}
L\left(w^{\prime}\right) & =L(R I(L I(u, b), a)) & & \text { def. of } w^{\prime} \\
& =L(L I(R I(u, a), b)) & & \text { induc., } H(u)<n \\
& =L(L I(a[x, y], b)) & & \\
& =L(a[x, L I(y, b)]) & & a \prec b \\
& =x & & \\
& =L(w) & & w=d[x, y]
\end{aligned}
$$

and

$$
\begin{aligned}
R\left(w^{\prime}\right) & =R(R I(L I(u, b), a)) & & \text { def. of } w^{\prime} \\
& =R(L I(R I(u, a), b)) & & \text { induc., } H(u)<n \\
& =R(L I(a[x, y], b)) & & \\
& =R(a[x, L I(y, b)]) & & a \prec b \\
& =L I(y, b) & & \\
& =L I(R(w), b) & & w=d[x, y]
\end{aligned}
$$

So

$$
a[L(w), c[L I(R(w), b), v]]=a\left[L\left(w^{\prime}\right), c\left[R\left(w^{\prime}\right), v\right]\right],
$$

and therefore $L I(R I(t, a), b)=R I(L I(t, b), a)$.

Theorem 3.3 Let $s$ be a sequence over $K$. Then $L T(s)=R T(\operatorname{rev}(s))$.

Proof (By strong induction over sequence lengths.) Base case: If $s=a_{1}$ and therefore $|s|=1$, then $\operatorname{LT}(s)=a_{1}[\perp, \perp]=R T(s)$.

Induction step: Assume that the claim holds for all sequences $s$ such that $|s|<n$. In other words, $L T(s)=R T(\operatorname{rev}(s))$ for all sequences $s$ such that $|s|<n$. Consider $s=a_{1} a_{2} \ldots a_{n}$.

$$
\begin{aligned}
& L T(s) \\
& =\operatorname{LI}\left(\operatorname{LT}\left(a_{1} \ldots a_{n-1}\right), a_{n}\right) \\
& =L I\left(R T\left(a_{n-1} \ldots a_{1}\right), a_{n}\right) \\
& =\operatorname{LI}\left(R I\left(R T\left(a_{n-1} \ldots a_{2}\right), a_{1}\right), a_{n}\right) \\
& =R I\left(L I\left(R T\left(a_{n-1} \ldots a_{2}\right), a_{n}\right), a_{1}\right) \quad \text { Lemma } 3.2 \\
& =R I\left(L I\left(L T\left(a_{2} \ldots a_{n-1}\right), a_{n}\right), a_{1}\right) \quad(* *) \\
& =R I\left(L T\left(a_{2} \ldots a_{n}\right), a_{1}\right) \\
& =R I\left(R T\left(a_{n} \ldots a_{2}\right), a_{1}\right) \\
& =R T\left(a_{n} \ldots a_{1}\right) \\
& =R T(\operatorname{rev}(s))
\end{aligned}
$$

(The justification for steps $(*),(* *)$, and $(* * *)$ is based on induction: $\left|a_{1} \ldots a_{n-1}\right|<n$, $\left|a_{n-1} \ldots a_{2}\right|<n$, and $\left|a_{2} \ldots a_{n}\right|<n$.)

The theorem just proven has important consequences: Any tree shape possible with leaf insertion is also possible with root insertion. Also, for a given tree $t$, the number of sequences $s$ and number of sequences $s^{\prime}$ of keys, such that $R T(s)=t=L T\left(s^{\prime}\right)$, are equal.

In the final result we show the equivalence of top-down and bottom-up root insertion.
Theorem 3.4 Let $s=a_{1} a_{2} \ldots a_{n}$ be a sequence of distinct keys. Then $R T(s)=R T^{\text {top }}(s)$ and $R C(s)=R C^{t o p}(s)$.

Proof Theorem 3.3 states that $R T(s)=$ $\operatorname{LT}(\operatorname{rev}(s))$, and we know from [9] that $R T^{\text {top }}(s)=\operatorname{LT}(\operatorname{rev}(s))$, and therefore $R T(s)=R T^{t o p}(s)$. By Definitions 2.2 and 2.3,

$$
R C(s)=C\left(R T\left(a_{1} \ldots a_{n-1}\right), a_{n}\right)+R C\left(a_{1} \ldots a_{n-1}\right)
$$

and

$$
\begin{aligned}
& R C^{t o p}(s) \\
& =C\left(R T^{t o p}\left(a_{1} \ldots a_{n-1}\right), a_{n}\right)+R C^{t o p}\left(a_{1} \ldots a_{n-1}\right)
\end{aligned}
$$

for $n \geq 2$. Since $R T\left(a_{1} \ldots a_{n-1}\right)=$ $R T^{t o p}\left(a_{1} \ldots a_{n-1}\right)$, it follows by induction that $R C(s)=R C^{t o p}(s)$.

In the remainder of the paper we will only consider bottom-up root insertion, and will simply refer to it as root insertion.

\section{WORST-CASE COST OF ROOT INSER- TION}

We define the worst-case cost of root insertion as $W_{n}^{r}:=\max \{R C(s)\}_{|s|=n}$. Our analysis of the worst-case cost of root insertion is based on expressing $R C(s)$ in terms of $R C(\tilde{s})$, where we obtain $\tilde{s}$ from $s$ by removing two elements from $s$. From this recurrence we derive an upper bound for $W_{n}^{r}$, and finally we construct a sequence for which the upper bound is reached. The proof of the next lemma contains no deep insight, but it is technical in nature. We will in fact only provide a sketch of the proof.

Lemma 4.1 Suppose $|s| \geq 3$. Then there is a sequence $\tilde{s}$, that is obtained from $s$ by removing two of the keys, and keeping the other keys in $s$ in their respective order, such that $R C(s) \leq R C(\tilde{s})+|s|+1$.

Proof Let $s=a_{1} a_{2} \ldots a_{n}$. We consider seven cases that occur when considering the structure of $R T\left(a_{1} \ldots a_{n}\right)$. In case 7 we consider the situation where $R T\left(a_{1} \ldots a_{n}\right)$ contains a node, such that this node has a non-empty left subtree and a non-empty right subtree. All other scenarios are covered by case 1 through case 6 . Also, case 1 and case 2 are symmetric cases, and similarly for cases 3 and 4, and cases 5 and 6 . In cases $1-6$, we define $\tilde{s}$ to be $a_{1} a_{2} \ldots a_{n-2}$.

Case 1: $a_{i} \prec a_{n-1} \prec a_{n}$ for $i=1, \ldots, n-2$. Let $\tilde{s}=a_{1} a_{2} \ldots a_{n-2}$. From the definition of $R C(s)$ we have that $R C(s)=R C(\tilde{s})+$ $C\left(R T(\tilde{s}), a_{n-1}\right)+C\left(R T\left(a_{1} a_{2} \ldots a_{n-1}\right), a_{n}\right)$. But $C\left(R T(\tilde{s}), a_{n-1}\right) \leq n-2$, since 


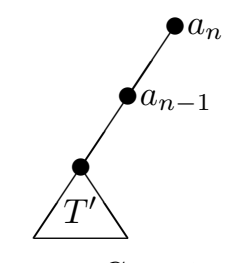

Case 1

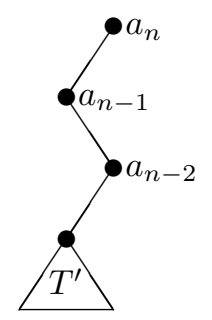

Case 3

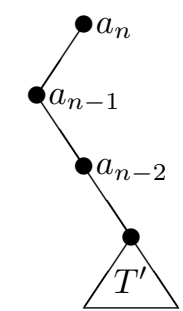

Case 5

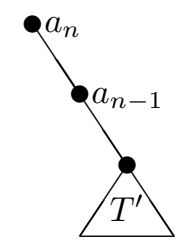

Case 2

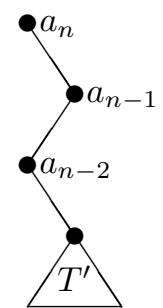

Case 4

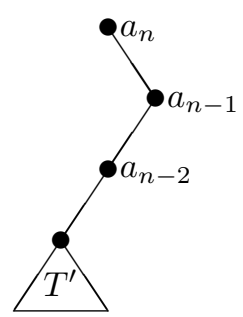

Case 6

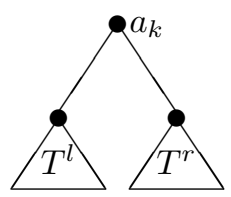

Case 7

Figure 3: The possibilities for the structure of $R T\left(a_{1} \ldots a_{n}\right)$ and $R T\left(a_{1} \ldots a_{k}\right)$, considered in the proof of Lemma 4.1 .

$|\tilde{s}|=n-2$. Also $C\left(R T\left(a_{1} a_{2} \ldots a_{n-1}\right), a_{n}\right)=$ $C\left(L T\left(a_{n-1} a_{n-2} \ldots a_{1}\right), a_{n}\right)=1$, since $a_{n}$ is only compared to $a_{n-1}$ when inserting $a_{n}$ into $R T\left(a_{1} a_{2} \ldots a_{n-1}\right)=\operatorname{LT}\left(a_{n-1} a_{n-2} \ldots a_{1}\right)$. Thus, $R C(s) \leq R C(\tilde{s})+(n-2)+1 \leq R C(\tilde{s})+|s|+1$.

Case 2: $a_{i} \succ a_{n-1} \succ a_{n}$ for $i=1, \ldots, n-2$. The argument is similar to Case 1 .

Case 3: $a_{i} \prec a_{n-2}$ for $i=1, \ldots, n-3, a_{i} \succ a_{n-1}$ for $i=1, \ldots, n-2$, and $a_{i} \prec a_{n}$ for $i=1, \ldots, n-1$. Let $\tilde{s}=a_{1} a_{2} \ldots a_{n-2}$. From the definition of $R C(s)$ we have that $R C(s)=R C(\tilde{s})+$ $C\left(R T(\tilde{s}), a_{n-1}\right)+C\left(R T\left(a_{1} a_{2} \ldots a_{n-1}\right), a_{n}\right)$. But $C\left(R T(\tilde{s}), a_{n-1}\right) \leq n-2$, since $|\tilde{s}|=n-2$. Also, $C\left(R \bar{T}\left(a_{1} a_{2} \ldots a_{n-1}\right), a_{n}\right)=$ $C\left(L T\left(a_{n-1} a_{n-2} \ldots a_{1}\right), a_{n}\right)=2$, since $a_{n}$ is only compared to $a_{n-1}$ and $a_{n-2}$ when inserting $a_{n}$ into $R T\left(a_{1} a_{2} \ldots a_{n-1}\right)=L T\left(a_{n-1} a_{n-2} \ldots a_{1}\right)$. Thus, $R C(s) \leq R C(\tilde{s})+(n-2)+2 \leq R C(\tilde{s})+|s|+1$.

Case 4: $a_{i} \succ a_{n-2}$ for $i=1, \ldots, n-3, a_{i} \prec a_{n-1}$ for $i=1, \ldots, n-2$, and $a_{i} \succ a_{n}$ for $i=1, \ldots, n-1$. Similar to case 3 .

Case 5: $a_{i} \succ a_{n-2} \succ a_{n-1}$ for $i=1,2, \ldots, n-3$, and $a_{i} \prec a_{n}$ for $i=1, \ldots, n-1$. Let $\tilde{s}=a_{1} a_{2} \ldots a_{n-2}$. From the definition of $R C(s)$ we have that $R C(s)=R C(\tilde{s})+$ $C\left(R T(\tilde{s}), a_{n-1}\right)+C\left(R T\left(a_{1} a_{2} \ldots a_{n-1}\right), a_{n}\right)$. But $C\left(R T(\tilde{s}), a_{n-1}\right)=1$, since $a_{n}$ is only compared to $a_{n-1}$ when inserting $a_{n}$ into $R T\left(a_{1} a_{2} \ldots a_{n-1}\right)=\operatorname{LT}\left(a_{n-1} a_{n-2} \ldots a_{1}\right)$. Also, $\quad C\left(R T\left(a_{1} a_{2} \ldots a_{n-1}\right), a_{n}\right) \quad=$ $C\left(L T\left(a_{n-1} a_{n-2} \ldots a_{1}\right), a_{n}\right) \leq n-1 . \quad$ Thus, $R C(s) \leq R C(\tilde{s})+(n-1)+1 \leq R C(\tilde{s})+|s|+1$.

Case 6: $a_{i} \prec a_{n-2} \prec a_{n-1}$ for $i=1,2, \ldots, n-3$, and $a_{i} \succ a_{n}$ for $i=1, \ldots, n-1$. Similar to case 5 .

Case 7: There exists a positive integer $k$ with $3 \leq$ $k \leq n$, such that $a_{l} \prec a_{k}$ for some $l \in\{1,2, \ldots k-$ $1\}$, and $a_{r} \succ a_{k}$ for some $r \in\{1,2, \ldots k-1\}$. We may assume that $a_{l}$ and $a_{r}$ are leaf nodes in the subtrees $T^{l}$ and $T^{r}$ that are indicated in case 7 in Figure 3. In order to simplify the argument in this case, we assume that $k$ is as small as possible such that the root of $R T\left(a_{1} \ldots a_{k}\right)$ has a non-empty left and right subtree. We need the following notation in the remainder of this proof. For $1 \leq j \leq n$, denote by $s_{j}$ the sequence $a_{1} \ldots a_{j}$. Also, for $j \geq k$ let $\bar{s}_{j}$ be the sequence $s_{j}$ with $a_{l}$ and $a_{r}$ deleted.

We let $\tilde{s}$ be the sequence $s$ with $a_{l}$ and $a_{r}$ deleted. Let $D:=R C(s)-R C(\tilde{s})$. We need to show that $D \leq(n+1)$. Since $D=$ $\alpha+\beta$, with $\alpha:=R C\left(s_{k}\right)-R C\left(\bar{s}_{k}\right)$ and $\beta:=$ $\sum_{i=k}^{n-1} C\left(\operatorname{LT}\left(\operatorname{rev}\left(s_{i}\right)\right), a_{i+1}\right)-C\left(\operatorname{LT}\left(\operatorname{rev}\left(\bar{s}_{i}\right)\right), a_{i+1}\right)$, it is enough to show that $\alpha \leq(k+1)$ and $\beta \leq$ $(n-k)$. We show that $\beta \leq(n-k)$ and leave 


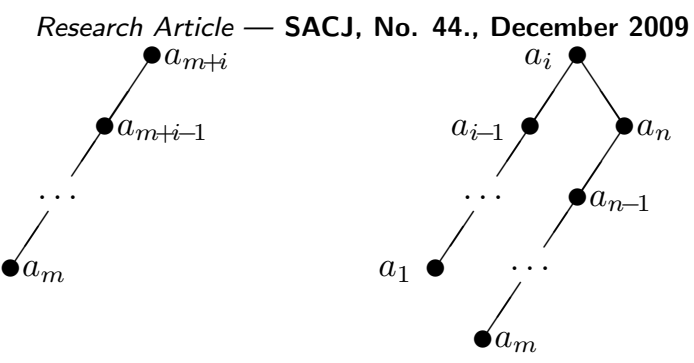

it to the reader as an easy but tedious exercise to verify that $\alpha \leq(k+1)$. We show that each term $\left[C\left(\operatorname{LT}\left(\operatorname{rev}\left(s_{i}\right)\right), a_{i+1}\right)-C\left(\operatorname{LT}\left(\operatorname{rev}\left(\bar{s}_{i}\right)\right), a_{i+1}\right)\right]$ in $\beta$ is at most 1 , and therefore that $\beta \leq n-k$. This follows from the following two observations on the trees $L T\left(\operatorname{rev}\left(s_{i}\right)\right)$ and $L T\left(\operatorname{rev}\left(\bar{s}_{i}\right)\right)$ for $k \leq i \leq$ $(n-1)$.

- $a_{l}$ and $a_{r}$ are leave nodes in $L T\left(\operatorname{rev}\left(s_{i}\right)\right)$, and once we remove these leave nodes from $L T\left(\operatorname{rev}\left(s_{i}\right)\right)$, the trees $\operatorname{LT}\left(\operatorname{rev}\left(s_{i}\right)\right)$ and $L T\left(\operatorname{rev}\left(\bar{s}_{i}\right)\right)$ are identical;

- any path from the root to a leaf in $L T\left(\operatorname{rev}\left(s_{i}\right)\right)$ contains at most one of $a_{l}$ or $a_{r}$.

From these two observations it follows that inserting $a_{i+1}$ in $R T\left(s_{i}\right)$ will be at most one comparison more expensive than inserting $a_{i+1}$ in $R T\left(\bar{s}_{i}\right)$.

Lemma 4.2 Let $n>1$. Then $W_{n}^{r} \leq n(n / 4+1)-$ $2-\alpha$ where $\alpha=0$ if $n$ is even and $\alpha=1 / 4$ if $n$ is odd.

Proof It is easy to verify that $W_{1}^{r}=0, W_{2}^{r}=1$, and $W_{3}^{r}=3$. Using these values and the previous lemma, we have that

$W_{n}^{r} \leq(n+1)+(n-1)+\cdots+5+W_{2}^{r}=n(n / 4+1)-2$

when $n$ is even, and

$$
\begin{aligned}
W_{n}^{r} & \leq(n+1)+(n-1)+\cdots+6+W_{3}^{r} \\
& =n(n / 4+1)-5 / 4
\end{aligned}
$$

when $n$ is odd.

Theorem 4.3 Let $n>1$. Then $W_{n}^{r}=n(n / 4+$ 1) $-2-\alpha$ where $\alpha=0$ if $n$ is even and $\alpha=1 / 4$ if $n$ is odd.

Proof From Lemma 4.2 we know that $n(n / 4+1)-$ $2-\alpha$ is an upper bound for $W_{n}^{r}$ when $n>1$. All that remains is to show that the bound is reached for every $n$. Consider the $n$ keys $a_{1} \prec a_{2} \prec \cdots \prec a_{n}$ and the sequence $s=a_{m} a_{m+1} \ldots a_{n} a_{1} a_{2} \ldots a_{m-1}$ where $m=\lfloor n / 2\rfloor+1$. Let $k=n-m+1$. The following table shows the cost of building the root insertion tree $R T(s)$ :

\begin{tabular}{cccc}
\hline Insert nr. & Key & Resulting tree & Cost \\
$i$ & $a$ & $t_{i}=R I\left(t_{i-1}, a\right)$ & $C\left(t_{i-1}, a\right)$ \\
\hline 1 & $a_{m}$ & $a_{m}[\perp, \perp]$ & 0 \\
2 & $a_{m+1}$ & $a_{m+1}\left[t_{1}, \perp\right]$ & 1 \\
3 & $a_{m+2}$ & $a_{m+2}\left[t_{2}, \perp\right]$ & 1 \\
$\vdots$ & & & \\
$k$ & $a_{n}$ & $a_{n}\left[t_{k-1}, \perp\right]$ & 1 \\
$k+1$ & $a_{1}$ & $a_{1}\left[\perp, t_{k}\right]$ & $k$ \\
$k+2$ & $a_{2}$ & $a_{2}\left[u_{1}, t_{k}\right]$ & $k+1$ \\
$k+3$ & $a_{3}$ & $a_{3}\left[u_{2}, t_{k}\right]$ & $k+1$ \\
$\vdots$ & & & \\
$n$ & $a_{m-1}$ & $a_{m}\left[u_{m-1}, t_{k}\right]$ & $k+1$ \\
\hline
\end{tabular}

Figure 4: Intermediary trees of the worst-case example in Theorem 4.3

where $t_{0} \quad=\quad \perp$ and $u_{r}=$ $a_{r}\left[a_{r-1}\left[\ldots a_{2}\left[a_{1}[\perp, \perp], \perp\right] \ldots, \perp\right], \perp\right] . \quad$ Figure 4 shows the resulting trees after the $i$-th insertion for $1 \leq i \leq k$ (on the left) and after the $(k+i)$-th insertion for $1 \leq i<m$ (on the right). Adding the numbers in the rightmost column of the table yields the desired result.

Although we shall not prove it, for $n=2$ both possible sequences produce the worst-case result. For $n=3$ and $n=4$ there are four such sequences, and when $n \geq 5$ there are eight sequences when $n$ is even, and sixteen when $n$ is odd.

\section{EXPERIMENTAL RESULTS}

In this section we provide experimental results that will provide the impetus for future investigations. We will not state the various obvious but interesting questions that can be asked by considering these experimental results. The results for root insertion were obtained by a brute-force approach of considering all $n$ ! sequences of length $n$, and counting for each sequence the number of comparisons required for root insertion.

Even though a brute-force approach is sufficient to obtain our experimental results for leaf insertion, we briefly describe an inductive method that can be used to obtain the cost distribution, in terms of number of comparisons, for inserting $n$ keys in a search tree by using leaf insertion. Although this result is most probably well-known, we could not find an appropriate reference. The reasoning required to obtain the result is more or less the same argument that is used to show that the average cost, $A_{n}^{\ell}$, to construct a leave insertion tree with $n$ keys is given by the recurrence $A_{n}^{\ell}=n-1+1 / n \sum_{1<k<n}\left(A_{k-1}^{\ell}+A_{n-k}^{\ell}\right)$. See for example [3], section 5.7, for a discussion of this result. For each $n \in\{1,2,3, \ldots\}$, let $L_{n}(z)$ be the polynomial with the coefficient of $z^{m}$ equal to the number of sequences of length $n$ for which the cost of constructing the leaf insertion tree is equal to $m$. For example, $L_{1}(z)=1=1 z^{0}$, since there is one sequence of length 1 and the cost of constructing 
the leaf insertion tree from this sequence is 0 . As a notational convenience, we define $L_{0}(z)$ to be 1 . We have for example that $L_{2}(z)=2 z$, since we have 2 sequences of length 2 and the cost of constructing a tree by leaf insertion from any of these two sequences is equal to 1 . Also, $L_{3}(z)=2 z^{2}+4 z^{3}$, since we have 2 sequences of length 3 for which the cost is 2 , and 4 sequences for which the cost is 3 . Note that the sum of the coefficients of $L_{n}(z)$ is equal to $n$ !, since we have $n$ ! sequences of length $n$. The polynomials $L_{n}(z)$ can also be defined recursively as follows: Let $n \geq 0$, then $L_{n+1}(z)=$ $z^{n}\left[\sum_{i=0}^{n}\left(\begin{array}{c}n \\ i\end{array}\right) L_{i}(z) L_{n-i}(z)\right]$. Thus we have for example that $L_{4}(z)=z^{3}\left(L_{0}(z) L_{3}(z)+3 L_{1}(z) L_{2}(z)+\right.$ $\left.3 L_{2}(Z) L_{1}(z)+L_{3}(z) L_{0}(z)\right)=12 z^{4}+4 z^{5}+8 z^{6}$. Therefore, if we consider the 24 sequences of length 4 , for 12 sequences the cost of constructing a leaf insertion tree is 4 , for 4 sequences the cost is 5 and for 8 sequences the cost is 6 . Similarly, $L_{5}(z)=$ $16 z^{10}+8 z^{9}+24 z^{8}+32 z^{7}+40 z^{6}$. The logic behind the formula for $L_{n+1}(z)$ is simple. A tree with $(n+1)$ keys, consists of a root and a left subtree of size $i$ and a right subtree of size $(n-i)$, for some $i$ between 1 and $n$. For any sequence $a_{1} \ldots a_{n+1}$ we select the $i$ positions from $2, \ldots, n+1$ that will contain the keys of the left subtree. This can be done in $\left(\begin{array}{c}n \\ i\end{array}\right)$ ways. The product $L_{i}(z) L_{n-i}(z)$ has terms $c z^{m}$, where $c$ is the number of pairs of sequence $\left(s_{1}, s_{2}\right)$, where the length of $s_{1}$ is $i$ and the cost of constructing a leaf insertion tree from $s_{1}$ is $j$, and the length of $s_{2}$ is $n-i$ and the cost of constructing a leaf insertion tree from $s_{2}$ is $m-j$. The additional term $z^{n}$, preceding $\left[\sum_{i=0}^{n}\left(\begin{array}{c}n \\ i\end{array}\right) L_{i}(z) L_{n-i}(z)\right]$, is required since each key added to the left or right subtree will require one more comparison to be inserted in a tree with $(n+1)$ keys, than if it were simply inserted in the left or right subtree on its own.

In the table below we list for sequence lengths $n=2$ to $n=13$, the percentage of sequences for which we need fewer comparisons (in column " $L<$ $R$ "), the same number of comparisons (in column " $L=R$ "), and more comparisons (in column " $L>$ $R$ ") for leaf insertion than for root insertion.

In Figure 5 the cost distributions of leaf and root insertion, for sequence lengths $n=6$ to $n=13$, are plotted. In each case, a point $(a, b)$ on a graph indicates that there are $b$ sequences, of length $n$, for which $a$ comparisons are required to construct the search tree. The solid and dotted lines represent leaf and root insertion, respectively. It is interesting to note that the graphs are almost smooth and symmetric for root insertion, but jagged and not symmetric for leaf insertion.

\begin{tabular}{cccc}
\hline$n$ & $L<R$ & $L=R$ & $L>R$ \\
\hline 2 & 0.0000 & 1.0000 & 0.0000 \\
3 & 0.3333 & 0.3333 & 0.3333 \\
4 & 0.4167 & 0.2500 & 0.3333 \\
5 & 0.4500 & 0.2000 & 0.3500 \\
6 & 0.4750 & 0.1333 & 0.3917 \\
7 & 0.5099 & 0.1040 & 0.3861 \\
8 & 0.5160 & 0.0926 & 0.3915 \\
9 & 0.5225 & 0.0819 & 0.3956 \\
10 & 0.5312 & 0.0691 & 0.3997 \\
11 & 0.5342 & 0.0627 & 0.4031 \\
12 & 0.5366 & 0.0575 & 0.4059 \\
13 & 0.5392 & 0.0525 & 0.4083 \\
\hline
\end{tabular}

\section{CONCLUSION}

The main result in this paper states that in the worst case, $n(n / 4+1)-2-\alpha(\alpha=0$ for $n$ even, and $\alpha=1 / 4$ for $n$ odd) comparisons are required to build a binary search tree with $n$ distinct keys, using root insertion. We were rather surprised by the fact that we could not find a proof of this result in the literature.

\section{REFERENCES}

[1] G. M. Adelson-Velsky, E. M. Landis. An algorithm for the organization of information. Soviet Math., 3:1259-1263, 1962.

[2] A. D. Booth, A. J. T. Colin. On the efficiency of a new method of dictionary construction. Information and Control, 3:327-334, 1960.

[3] P. Flajolet, R. Sedgewick. An Introduction to the Analysis of Algorithms. Addison-Wesley, 1996.

[4] T. N. Hibbard. Some combinatorial properties of certain trees with applications to searching and sorting. Journal of the ACM, 9:13-28, 1962.

[5] A. T. Jonassen, D. E. Knuth. A trivial algorithm whose analysis isn't. Journal of Computer and System Sciences, 16:301-322, 1978.

[6] D. E. Knuth. Sorting and Searching, Volume 3 of The Art of Computer Programming. AddisonWesley, 1973.

[7] R. Sedgewick. Algorithms in Java, Parts 1-4, Addison-Wesley Professional, 3rd edition, 2003.

[8] D. Sleator, R. E. Tarjan. Self-adjusting binary trees. Proc. 15th Symp. Theory of Computing, 235245, 1983.

[9] C. J. Stephenson. A method for constructing binary search trees by making insertions at the root. International Journal of Computer and Information Sciences, 9:15-29, 1980. 

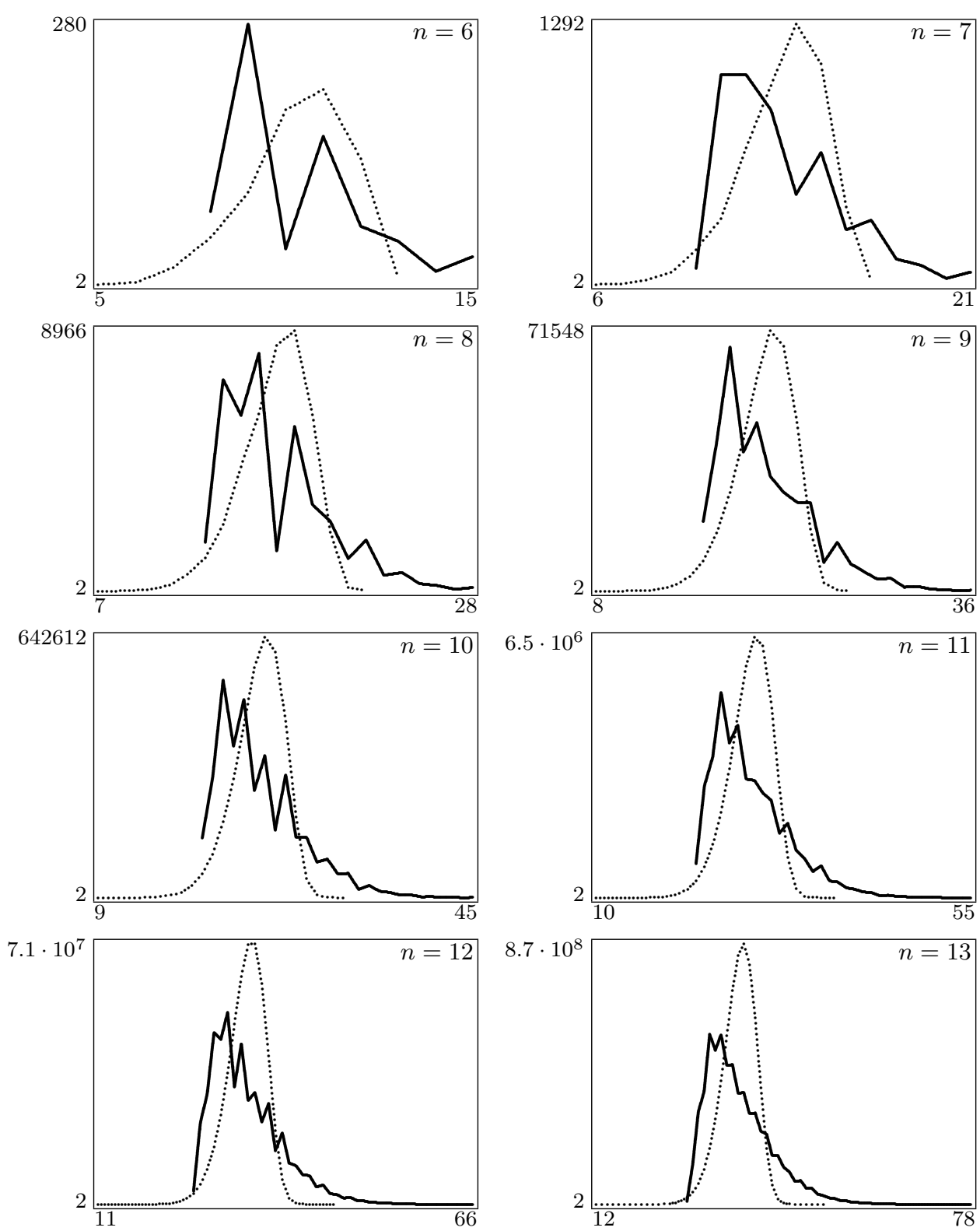

Figure 5: Cost distribution of leaf and root insertion for sequence lengths $n=6$ to $n=13$. The solid and dotted lines represent leaf and root insertion, respectively. 